Original article

\title{
M-MODE LEFT VENTRICULAR ECHOCARDIOGRAPHIC INDICES OF TRYPANOSOMA BRUCEI INFECTED DOGS
}

\author{
E. S. AJIBOLA ${ }^{1}$, J. O. OYEWALE ${ }^{2} \&$ B. O. OKE ${ }^{2}$ \\ ${ }^{1}$ College of Veterinary Medicine, Federal University of Agriculture, \\ Abeokuta, Nigeria; ${ }^{2}$ Faculty of Veterinary Medicine, \\ University of Ibadan, Ibadan, Nigeria
}

\section{Summary}

Ajibola, E. S., J. O. Oyewale \& B. O. Oke, 2016. M-mode left ventricular echocardiographic indices of Trypanosoma brucei infected dogs. Bulg. J. Vet. Med., 19, No 4, 274-280.

This study is aimed at understanding the structural and functional changes that could occur in the left ventricle of dogs acutely infected with Trypanosoma brucei. Twenty clinically healthy adult dogs of both sexes with an average weight of $8.4 \mathrm{~kg}$ were used for the study. They were divided into infected and uninfected groups of fifteen and five dogs, respectively. Each dog was intra-peritoneally inoculated with $1 \mathrm{~mL}$ of phosphate buffered saline (PBS) diluted blood containing $1 \times 10^{6}$ of Lafenwa strain of T. brucei. M-mode echocardiogram of the left ventricle was obtained from right parasternal short axis view before and at sixteen days after infection. The internal dimension indices were recorded by the in-built caliper of an ultrasound machine and functional indices obtained using appropriate formula. In both systole and diastole, the left ventricular internal dimensional indices of infected dogs were significantly lower than values obtained for uninfected $\operatorname{dogs}(\mathrm{P}<0.05)$. In comparison to control dogs, the end diastolic volume (EDV) and left venticular mass (LVM) of infected dogs were significantly reduced $(\mathrm{P}<0.05)$. The ejection fraction $(\mathrm{EF})$ and fractional shortening $(\mathrm{FS})$ of infected dogs were not statistically different from control dogs $(\mathrm{P}>0.05)$. While the reduced end systolic volume index of infected dogs may be due to their smaller systolic ventricular dimension, LVM reduction in infected dogs may be attributable to cardiac muscle necrosis. The stability in both EF and FS of infected dogs may result from left ventricular compensatory efforts against sepsis induced myocardial depression. The preload and after load dependent nature of EF and FS make them unsuitable as candidate markers of myocardial dysfunction in acute canine trypanosomiasis

Key words: diameter, dog, echocardiogram, ventricle, volume

\section{INTRODUCTION}

According to the World Health Organization (WHO, 2005), the burden of heart disease is increasing rapidly and has constituted a public health problem throughout the African continent. Human African trypanosomiasis (HAT) is a neglected tropical disease affecting poor rural dwellers (Hasker et al., 2012). Therapeutic intervention in HAT patients may not be successful because many of them with 
cardiac form of the disease may also suffer from underlying heart ailment (McCarroll et al., 2015). The early detection of cardiac involvement in HAT is therefore desirable in order to reduce economic waste and mortality.

The increasing wave of rural-urban migration and the role of dogs as reservoir host of HAT is a major challenge to the control of the disease. The role of dogs as reservoir hosts in human trypanosomiasis is well documented (Matete, 2003; Njiru et al., 2004). While dogs could serve as a good model in understanding this disease in man, there is also the need to adequately diagnose the disease in the dog population if the disease is to be prevented or controlled in man.

A striking similarity in pathogenesis and pathology caused by $T$. brucei in infected dogs and humans has been noticed. In both HAT and canine African trypanosomiasis (CAT), cardiomyopathies leading to congestive heart failure have been reported (Morrison et al., 1981; Blum et al., 2007). The use of T. brucei infected dog as a model in understanding the role of the heart in the pathogenesis of HAT is therefore a priority for researchers and clinicians.

Several attempts involving the application of electrocardiography and cardiac biomarkers in understanding heart involvement in both HAT and CAT have been reported (Blum et al., 2007; Ndungu et al., 2014). None of these approaches directly assesses cardiac function and size. The use of echocardiography, a technique that non-invasively determines cardiac function and size has not been utilised in understanding CAT and even HAT.

A major objective of this study would therefore be to investigate changes in left ventricular echocardiographic indices in T. brucei infected dogs. This work is also aimed at identifying potential echocardiographic markers of cardiac dysfunction in acute $T$. brucei infection of dogs.

\section{MATERIALS AND METHODS}

Institutional approval for this study was granted by the Animal Ethics Committee of the College of Veterinary Medicine, Federal University of Agriculture, Abeokuta, Nigeria.

Twenty adult dogs sourced randomly from local breeders in Abeokuta were used for this study. The dogs were of mixed sexes, ranging in weight between 6 and $15 \mathrm{~kg}$. They were acclimatised to laboratory conditions for two weeks and screened against trypanosome species and other haemoparasites. Each of the dogs was marked on the head with an indelible marker for ease of identification. The dogs received all routine vaccinations against common dog diseases like canine distemper, leptospirosis, hepatitis, parvovirus enteritis and were all certified free from heartworm. Cardiac health of the dogs was assessed by auscultation and ECG examination. Animals with cardiac abnormalities were excluded from the study. They were kept in a netted room of $100 \mathrm{~m}^{2}$ measuring $10 \times 10 \mathrm{~m}$ and fed twice daily on cooked local foods and fish diet.

The twenty dogs was divided into two groups: infected and uninfected control. The infected group comprised 15 dogs and the uninfected control group - 5 dogs.

Each infected dogs received $1 \mathrm{~mL}$ of $1 \times 10^{6}$ of the parasite in phosphate buffered saline (PBS) intra-peritoneally (Ajibola \& Oyewale, 2014), while the uninfected groups received $1 \mathrm{~mL}$ of normal saline through the same route. Echocardiogram was obtained before and at 16 days post infection when the parasite was expected to have invaded the heart tissue 
and caused parasitic myocarditis (Morrison et al., 1981)

A long and short axis para-sternal echocardiogram of each of the dogs was taken in standing position without anaesthesia. The echocardiographic examination was conducted with ultrasound scanner (Leidal Medical, England) with convex probe of frequency $5 \mathrm{MHz}$. An acoustic gel was applied at the interface between the transducer and echo window. A 2D guided M-mode measurements of the left ventricle at the level of papillary muscle were obtained from the short axis right parasternal view. The following indices were obtained using the in-built caliper of the echocardiograph: left ventricular internal diameter in diastole (LVIDd), left ventricular internal diameter in systole (LVIDs), interventricular septum thickness in diastole (IVSTd), interventricular septum wall thickness in systole (IVSTs), posterior wall thickness in diastole (PWTd), posterior wall thickness in systole (PWTs). Left ventricular end diastolic and systolic measurements were taken at the largest and at the smallest dimensions between the interventricular septum and the left ventricular free wall respectively. Five individual measurement of each variable were obtained and averaged. All measurements were made according to standard convention.

The following indices were derived according to the method of Bonagura (1983):

Fractional shortening $(\mathrm{FS})=($ LVIDd LVIDs/LVIDd) $\times 100$;

Ejection Fraction $(\mathrm{EF})=\mathrm{LVIDd}^{3}-$ $\mathrm{LVIDs}^{3} / \mathrm{LVIDd}^{3}$.

End diastolic volume (EDV) and end systolic volume (ESV) were derived from the formula of Teicholz et al (1976):

$\mathrm{EDV}=7(\mathrm{LVIDd})^{3} / 2.4+$ LVIDd;

$$
\mathrm{ESV}=7(\mathrm{LVIDs})^{3} / 2.4+\text { LVIDs. }
$$

The stroke volume (SV) in $\mathrm{cm}^{3}$ was calculated as EDV-ESV.

The left ventricular mass (LVM) was obtained using the formula of Troy et al., 1972:

LVM $=1.05(\text { LVIDd }+ \text { PWTd }+ \text { IVSTd })^{3}-$ $(\text { LVIDd })^{3}$.

The EDV, ESV, SV and LVM were all indexed to the body surface area (BSA) to obtain EDV-I, ESV-I, and SV-I respectively.

\section{Statistical analysis}

All data were expressed as mean \pm standard deviation. Differences in parameters between groups were evaluated using Student's t-test for independent samples at a level of significance $\mathrm{P}<0.05$. All analyses were done using SPSS version 17.

\section{RESULTS}

The results of this study (Table 1) showed that in both systole and diastole, left ventricular internal diameter, posterior wall thickness, interventricular septal thickness of dogs infected with $T$. brucei were significantly lower than in the control dogs $(\mathrm{P}<0.05)$. The indexed left ventricular mass (LVM-I) of infected dogs was significantly lower than the value obtained in uninfected controls.

As seen from Table 2, all studied cardiac function indices of infected dogs like fractional shortening (FS), ejection fraction $(\mathrm{EF})$, indexed end diastolic volume (EDV-I) and indexed stroke volume (SVI) were not different from values obtained from control dogs $(\mathrm{P}>0.05)$. The indexed end systolic volume (ESV-I) of infected dogs was significantly lower than the value obtained in control dogs $(\mathrm{P}<0.05)$. 
Table 1. Left ventricular internal dimension indices (mean $\pm \mathrm{SD})$ of control $(\mathrm{n}=5)$ and $T$. brucei infected dogs $(n=15)$.

\begin{tabular}{lccc}
\hline Left ventricular indices & Uninfected & Infected & P-value \\
\hline $\begin{array}{l}\text { Left ventricular internal diameter in diastole } \\
(\mathrm{mm})\end{array}$ & $25.87 \pm 7.56$ & $15.75 \pm 4.27$ & 0.015 \\
& & & \\
$\begin{array}{l}\text { Left ventricular internal diameter in systole } \\
(\mathrm{mm})\end{array}$ & $11.50 \pm 2.50$ & $7.50 \pm 2.64$ & 0.047 \\
$\begin{array}{l}\text { Posterior wall thickness in diastole }(\mathrm{mm}) \\
\text { Posterior wall thickness in systole }(\mathrm{mm})\end{array}$ & $12.37 \pm 2.87$ & $7.25 \pm 1.50$ & 0.002 \\
$\begin{array}{l}\text { Inter ventricular septal thickness in diastole } \\
(\mathrm{mm})\end{array}$ & $18.75 \pm 4.80$ & $11.75 \pm 1.89$ & 0.005 \\
$\begin{array}{l}\text { Inter ventricular septal thickness in systole } \\
(\mathrm{mm})\end{array}$ & $17.00 \pm 3.38$ & $11.75 \pm 3.94$ & 0.04 \\
Left ventricular mass $\left(\mathrm{g} / \mathrm{m}^{2}\right)$ & $275.50 \pm 141.06$ & $63.94 \pm 39.08$ & 0.04 \\
\hline
\end{tabular}

Table 2. Cardiac function indices (mean $\pm \mathrm{SD})$ of control $(\mathrm{n}=5)$ and $T$. brucei infected dogs $(\mathrm{n}=15)$.

\begin{tabular}{lccc}
\hline Cardiac function indices & Uninfected & Infected & P-value \\
\hline Ejection fraction & $0.90 \pm 0.06$ & $0.84 \pm 0.15$ & 0.38 \\
Fractional shortening (\%) & $52.39 \pm 12.73$ & $51.00 \pm 17.45$ & 0.87 \\
Indexed end diastolic volume $\left(\mathrm{m}^{2}\right)$ & $73.60 \pm 62.85$ & $21.16 \pm 13.25$ & 0.09 \\
Indexed end systolic volume $\left(\mathrm{m}^{2}\right)$ & $8.90 \pm 5.81$ & $2.15 \pm 1.28$ & 0.03 \\
Indexed stroke volume $\left(\mathrm{m}^{2}\right)$ & $64.68 \pm 58.48$ & $19.00 \pm 13.88$ & 0.17 \\
\hline
\end{tabular}

\section{DISCUSSION}

Although T. brucei infection did not appear to affect haemodynamic variables like SV, EDV, FS and EF, all ventricular internal dimension indices like LVIDd, LVIDs, PWTd, PWTd, IVSTd and IVSTs were reduced in infected dogs as reported for septicaemic dogs (Dickinson et al., 2007). The reduction in the LVM of infected dogs which may have been caused by a significant reduction in PWT, IVST and LVID in both diastole and systole has further laid credence to the report of cardiac muscle mass destruction by necrosis and infarction in T. brucei infection (Morrison et al., 1981).
In this study, depressed myocardial contractility envisaged is not evident because there was no significant difference in the FS and EF of control and infected dogs. The infected dogs also had EF and FS of above $40 \%$ and $20 \%$ respectively. EF of less than $40 \%$ and FS of less than $20 \%$ respectively has been proposed as a diagnostic criterion for dilated cardiomyopathy (DCM) in dogs (Dukes-McEwan, 2003). The systolic function indices (FS and EF) employed in this study are afterload dependent (Mahler et al., 1975), and so favorable loading conditions could mask the effects of depressed myocardial contractility which otherwise could produce a low EF and FS (Ross, 1983). In anaemic conditions, preload has been re- 
ported to increase as a result of decreased peripheral resistance and afterload decreased as a result of lowered blood viscosity. A favorable afterload and preload condition would most likely enhance myocardial performance in the face of myocardial depression often experienced in septic conditions like CAT. Often with time patients with sepsis induced myocardial depression have normal cardiac output. This depression is caused by various mechanism like aptosis of the cardiovascular centers in the medulla, mitochondrial dysfunction, and down regulation of adrenergic receptors (Rudiger \& Singer, 2007). In mild form of myocardial depression, normal cardiac pump function can often be maintained by compensatory mechanism. In a canine model of acute normovolemic anaemia echocardiographic evidence of left ventricular hyperdynamic response to anaemia has also been reported. It was found that the severely anaemic dogs had increased FS and EF.

At less than $30 \mathrm{~mL} / \mathrm{m}^{2}$ the ESV-I of both infected and control dogs are within the reference range for dogs (Freeman et al., 1996). The decreased ESV-I, rather than the expected increase in ESV-I of the infected dogs (Dickinson et al., 2007) could either be caused by favorable afterload condition or the reduced systolic volume of infected dogs. This reduction in ESV-I may have little or nothing to do with EDV-I and SV-I, both of which were not affected by the infection.

Studies conducted on sheep and cattle have shown that $T$. congolense caused a significant increase in blood and plasma volume of the infected animals (Dargie et al., 1979; Katunguka-Rwakishaya et al., 1992). Recently, evidence of increased plasma volume has also been found in canine babesiosis (Schetters et al., 1997). Although plasma volume determination was not included in this study, a possible increase in plasma volume of the infected dogs will have a dramatic effect on the haemodynamic indices. The expected blood volume increase in the infected dogs could cause hypovolemic anaemia, reduced peripheral resistance, increased preload which may ultimately lead to an increase in EDV, SV and CO thereby neutralising the envisaged sepsis induced myocardial depression.

In conclusion, $T$. brucei infection in this study affected left ventricular chamber dimension without affecting the cardiac function indices like EF, FS, EDV-I, and SV-I. The compensatory left ventricular hyperdynamic response to anaemia and myocardial depression is possibly the reason for this.

In acute $T$. brucei infection, the use of functional indices like EF, FS as a diagnostic criteria of myocardial dysfunction is not recommended as they are both preload and after load dependent. The use of left ventricular internal dimension indices and LVM as diagnostic marker of acute canine trypanosomiasis is therefore proposed.

\section{REFERENCES}

Ajibola, E. S. \& J. O. Oyewale, 2014. Relationship between some serum electrolytes and electrocardiographic indices of Trypanosoma brucei infected dogs. Sokoto Journal of Veterinary Science, 14, 36-44.

Blum, J. A., C. Burri, C. Hatz, I. Kazumba, P. Mangoni \& M. J. Zellweger, 2007. Sleeping Hearts: the role of the heart in sleeping sickness (human African trypanosomiasis). Tropical Medicine and International Health, 12, 1422-1432.

Bonagura, J. D., 1983. M-mode echocardiography: Basic principles. Veterinary Clinics of North America: Small Animal Practice, 13, 299-320. 
Dargie, J. D., M. Murray, W. R. Grimshaw \& W. I. M. McIntrye, 1979. Bovine trypanosomiasis: Red cell kinetics of Ndama and Zebu cattle infected with Trypanosoma congolense. Parasitology, 78, 271-286.

Dickinson, A. E., E. A. Rozanski \& J. E. Rush, 2007. Reversible myocardial depression associated with sepsis in a dog. Journal of Veterinary Internal Medicine, 21, 11171120.

Dukes-Mc Ewans, J., M. Borgarelli, A. Tidholm, A. Vollmar \& J. Haggstron, 2003. Proposed guideline for the diagnosis of canine idiopathic dilated cardiomyopathy. Journal of Veterinary Cardiology, 5, 7-9.

Freeman, L. M., K. M. Michel, D. Brown, P. M. Kaplan, M. E. Stamoulis, S. L. Rosenthal, B. W. Keene \& J. E. Rush, 1996. Idiopathic dilated cardiomyopathy in Dalmatians: Nine cases (1990-1995). Journal of the American Veterinary Medical Association, 209, 1592-1596.

Hasker, E., P. Lutumba, F. Chappuis, V. Kande, J. Potet, A. De Weggheleire, C. Kambo, E. Depoortere, B. Pecoul \& M. Boelaert, 2012. Human African trypanosomiasis in the Democratic Republic of the Congo: A looming emergency. PLOS Neglected Tropical Diseases, 6, e1950. doi: 10.1371/journal.pntd.0001950

Katunguka-Rwakishaja, E., M. Murray \& P. H. Holmes, 1992. Pathophysiology of ovine trypanosomiasis: Ferrokinetics and erythrocyte survival studies. Research in Veterinary Science, 53, 80-86.

Mahler, F., J. Ross, R. A. O’ Rourke \& J. W. Covell, 1975. Effects of load changes in preload, afterload, and inotropic state on ejection and isovolumic phase measures of contractility in the conscious dog. American Journal of Cardiology, 35, 626-634.

Matete, G. O., 2003. Occurrence, clinical manifestation and the epidemiological implications of naturally occurring canine trypanosomiasis in Western Kenya. Onderstepoort Journal of Veterinary Research, 70, 317-323.
McCarroll, C. S., C. L. Rossor, L. R. Morrison, L. J. Morrison \& C. M. Loughray, 2015. A preclinical animal model of Trypanosoma brucei demonstrating cardiac dysfunction. PLOS Neglected Tropical Diseases, 9, e0003811.doi;10.1371/journal.pntd.000381.

Morrison, W. I., M. Max, P. D. Sayer \& J. M. Preston, 1981. The pathogenesis of experimentally induced Trypanosoma brucei infection in the dog: Tissue and organ damage. American Journal of Pathology, 102, 168-181.

Ndungu, J. M, N. A. McEwan, F. W. Jennings \& M. Murray, 1991. Cardiac damage in dogs infected with T. brucei: Clinical and eectrocardiographic features. Journal of Small Animal Practice, 32, 579-584.

Njiru, Z. K., K. Ndungu, G. Matete, J. M. Ndungu \& W. C. Gibson, 2004. Detection of Trypanosoma brucei rhodesiense in animals from sleeping sickness foci in East Africa using the serum resistance associated (SRA) gene. Acta Tropica, 90, 249254.

Ross, J. Jr., 1983. Cardiac function and myocardial contractility: A perspective. Journal of American College of Cardiology, $\mathbf{1}$, 52-62.

Rudiger, N. \& M. Singer, 2007. Mechanisms of sepsis-induced cardiac dysfunction. Critical Care, 35, 1599-1608.

Schetters, T. P. M., K. Moubri, E. Precigout, J. Kleuskens, N. C. Scholtes \& A. Gorenflot, 1997. Different Babesia canis isolates, different diseases. Parasitology, 115, 485493.

Teichholz, L. E., T. K. Kreulen, M. V. Herman \& R. Gorlin, 1976. Problems in echocardiographic volume determinations: Echocardiographic angiographic correlations in the presence of absence of synergy, American Journal of Cardiology, 37, 7-11.

Troy, B. L., J. Pombo \& C. E. Rackley, 1972. Measurement of left ventricular wall thickness and mass by echocardiography. Circulation, 45, 602-611. 
M-mode left ventricular echocardiographic indices of Trypanosoma brucei infected dogs

WHO Regional Committee for Africa, 2005. Cardiovascular diseases in the African region: Current situation and perspectives Report of the regional director. file://C:/Users/PC2/Desktop/AFR\%20RC 55-12\%20Cardiovascular\%20Diseases $\%$ 20Latest-5b.pdf (21 December 2015 date last accessed).

Paper received 05.10.2015; accepted for publication 13.11.2015

\section{Correspondence:}

E. S. Ajibola

College of Veterinary Medicine,

Federal University of Agriculture,

Abeokuta, Nigeria,

e-mail: esajibola@yahoo.com 